Insulin resistance

\section{Tissue-specific insulin resistance}

\section{A G Unnikrishnan}

\section{Helping to understand the metabolic syndrome}

$\mathrm{T}$ he combination of abnormal glucose tolerance, central adiposity, dyslipidaemia, hypertension, and coronary artery disease is together referred to as the metabolic syndrome or the insulin resistance syndrome. ${ }^{1} \mathrm{~A}$ sedentary lifestyle, polygenic inheritance, and abnormal intrauterine programming are but some of the hypotheses put forward to explain the syndrome, the central underlying feature of which is insulin resistance. The exact genesis of the syndrome is not well understood.

Despite the well characterised links between insulin resistance and the components of the insulin resistance syndrome, the mechanism by which insulin resistance leads to the metabolic syndrome is still unclear. Recent studies suggest that the reason for the heterogeneity of the metabolic syndrome could be the differing, or sometimes opposing, effects of insulin in the different tissues of the body. ${ }^{2}$

There is a growing interest in animal models that knock out the insulin receptor or its action in specific tissues only..$^{2-5}$ For example knocking out insulin receptors in the adipose tissue will cause insulin resistance at the adipose tissue level, but normal insulin sensitivity in other tissues like liver and muscle. These "tissue"-specific knockout models result in different phenotypes depending upon the tissues where insulin receptors have been knocked out. Studies from the knockout models produce characteristic phenotypes very similar to certain classic clinical enigmas: for instance, why do some people have the metabolic syndrome without developing glucose intolerance? Why do only some subjects develop a fatty liver or non-alcoholic steatohepatitis (NASH) as part of the metabolic syndrome? Why do some type 2 diabetics have $\beta$-cell dysfunction (rather than insulin resistance) as the predominant abnormality?
To begin with, skeletal muscle insulin receptor knockout models generated fewer disturbances of glucose homoeostasis, despite severe insulin resistance. ${ }^{6}$ In other words, this resistance at the skeletal muscle but sensitivity in fat resulted in more nutrient storage as fat, resulting in visceral adiposity, with consequent dyslipidaemia, and cardiovascular risk, despite normal glucose tolerance (probably due to retained hepatic sensitivity to insulin). This could explain why some individuals have the metabolic syndrome and risk of heart disease without any glucose intolerance.

Even more interesting is the role of the liver. Liver insulin resistant knockout mice have been shown to develop diabetes. $^{7}$ Interestingly, this knockout can lead to progressive hepatic dysfunction, $^{7}$ and may explain why some subjects with metabolic syndrome eventually develop NASH, now considered a component of the insulin resistance syndrome.

It is well known that type 2 diabetes occurs due to a combination of $\beta$-cell dysfunction and insulin resistance. The mechanism of $\beta$-cell dysfunction remains unknown. Interestingly, an insulin resistant knockout model, at the level of the pancreatic $\beta$-cell, caused disordered insulin secretion and type 2 diabetes; thus insulin might feedback on the $\beta$-cell to modulate its own secretion, and insulin resistance at the $\beta$-cell level might affect this modulation. This is a fascinating hypothesis for explaining $\beta$-cell dysfunction in type 2 diabetes. ${ }^{8}$

These reports indicate that insulin resistance can no longer be considered as only a uniform systemic alteration. Conceptually, it is better to consider insulin resistance as being tissue-specific, and the ultimate phenotype of the individual depends on the sum total of the effects of the differing sensitivities of the differing tissues.
Almost all research into the genesis of the metabolic syndrome has not yielded a single, unifying theory, but only more and more heterogeneity. Therefore, it is now time to approach the problem from a fresh perspective. This focus on individual tissues rather than the entire individual could probably open up new avenues. For example subjects with hepatic insulin resistance may present with a phenotype that is distinct from those with resistance at the skeletal muscle. Already treatment strategies are being targeted at certain specific tissues-for instance, the predominantly hepatic level insulin resistance is combated by metformin. Is it too futuristic to predict that insulin resistant individuals could be classified as hepatic resistors, skeletal muscle resistors etc, and treatment strategies tailored to suit the site of resistance?

There is a need for a fresh perspective on the insulin resistance syndrome, the prevalence of which is now fast attaining epidemic proportions worldwide. The concept of tissue-specific insulin resistance could help to change the way we understand the metabolic syndrome, as well as open fascinating avenues for research into potential therapeutic interventions.

Postgrad Med J 2004;80:435.

doi: $10.1136 /$ pgmi.2004.023176

Correspondence to: $\operatorname{Dr}$ A G Unnikrishnan Department of Endocrinology, Amrita Institute of Medical Sciences, Cochin 682026, Kerala, India; unnikrishnanag@aimshospital.org

\section{REFERENCES}

1 Reaven GM. Role of insulin resistance in human disease. Diabetes 1988;37:1595-607.

2 Blüher M, Kahn BB, Kahn RC. Extended longevity in mice lacking the insulin receptor in adipose tissue. Science 2003;299:572-4.

3 Blüher M, Michael MD, Peroni OD, et al. Adipose tissue selective insulin receptor knockout protects against obesity and obesity-related glucose intolerance. Dev Cell 2002;3:25-38.

4 Barzilai N, Gupta G. Revisiting the role of fat mass in life extension induced by caloric restriction. J Gerontol Biol Sci 1999;54A:B89-96.

5 Hamann A, Flier JS, Lowell BB, et al. Decreased brown fat markedly enhances susceptibility to diet induced obesity, diabetes and hyperlipidemia. Endocrinology 1996;137:21-9.

$6 \mathrm{Kim}$ JK, Michael MD, Previs SF, et al. Redistribution of substrates to adipose tissue promotes obesity in mice with selective insulin resistance in muscle. J Clin Invest 2000;105:1791-7.

7 Michael MD, Kulkarni RN, Postic C, et al. Loss of insulin signaling in the hepatocytres leads to severe insulin resistance and progressive hepatic dysfunction. Mol Cell 2000;6:87-97.

8 Kulkarni RN, Bruning JC, Winnay JN, et al. Tissue specific knockout of the insulin receptor in pancreatic beta cells creates an insulin secretory defect similar to that in type 2 diabetes. Cell 1999;96:329-39. 